\title{
A New Method for Setting Futures Portfolios' Maintenance Margins: Evidence from Chinese Commodity Futures Markets
}

\author{
Chi Xie, ${ }^{1,2}$ Jiao-Jiao Yang, ${ }^{1}$ and Gang-Jin Wang ${ }^{1,2}$ \\ ${ }^{1}$ College of Business Administration, Hunan University, Changsha 410082, China \\ ${ }^{2}$ Center of Finance and Investment Management, Hunan University, Changsha 410082, China \\ Correspondence should be addressed to Gang-Jin Wang; wanggangjin@hnu.edu.cn
}

Received 4 August 2013; Revised 18 January 2014; Accepted 19 January 2014; Published 20 March 2014

Academic Editor: Zhijun Liu

Copyright (c) 2014 Chi Xie et al. This is an open access article distributed under the Creative Commons Attribution License, which permits unrestricted use, distribution, and reproduction in any medium, provided the original work is properly cited.

\begin{abstract}
The Chinese commodity futures markets neglect the existence of the risk hedge and diversification between futures contracts, thus leading to overcharge futures portfolio holders' maintenance margins. To this end, this paper proposes a new method, namely, the multivariate $t$-Copula-POT-PSRM method, which combines three models, that is, the multivariate $t$-Copula, the peaks over threshold (POT), and the power spectral risk measures (PSRM), to set futures portfolios' maintenance margins. In the empirical analysis, we first construct four kinds of futures portfolios and set their maintenance margins by using the new method. Then, we introduce two evaluation indicators, namely, the prudence index (PI) and the opportunity cost index (OCI), to assess the effectiveness of the proposed method. We also compare the outcomes of the two evaluation indicators of the new method with those of the widely used linear additive model. The empirical results show that the new method can, respectively, lower the OCI value of all four kinds of futures portfolios for the In-sample period and the Out-of-sample period without significantly reducing the PI value as against the traditional model, which implies that the proposed method can be used to balance security and investment efficiency in the futures market.
\end{abstract}

\section{Introduction}

As a means of controlling risk as well as a guarantee of fulfilling the contracts in futures trading, futures margins are indispensable to the futures market and even the entire capital market. According to the nature and role of futures margins, they can be classified into the clearing margin and the trading margin. The trading margin includes the initial margin and the maintenance margin. The initial margin is the minimum levels to open futures positions, while the maintenance margin refers to the minimum levels to carry futures positions. Setting maintenance margins runs through the whole course of futures trading, and the effectiveness of the maintenance margins greatly influences the stability and efficiency of the futures market.

Although the Chinese futures market is an emerging market, it has become the world's biggest commodity futures market since its turnover exceeded RMB 300 trillion in 2010. With the sustainable growth of the Chinese futures market, the international influence of the Chinese commodity futures exchanges is growing rapidly. There are three commodity futures exchanges in Chinese commodity futures markets, namely, the Dalian Commodity Exchange (DCE), the Shanghai Commodity Exchange (SHFE), and the Zhengzhou Commodity Exchange (ZCE). The Chinese commodity futures exchanges are in the initial period from the quantitative expansion to the qualitative leap nowadays, but also face with some challenges especially in setting maintenance margins. The three commodity futures exchanges only consider a single futures contract to set the maintenance margins, while ignoring the existence of the risk diversion and risk hedge between futures contracts. Meanwhile, they also neglect that holding multiple future contracts and investing in futures portfolios become the main choice for the futures investors. Actually, the commodity futures exchanges just linearly add the maintenance margins of all futures contracts when the maintenance margins are charged to the futures portfolio holder, which implies that there exists a high opportunity cost as well as a serious occupation of investment capitals. At the same time, the commodity futures exchanges cannot 
precisely describe the tail risk and choose a sound risk measure to estimate the risk of futures portfolios.

In setting futures portfolios' maintenance margins, the dependence structure between futures contracts and the overall risk of futures portfolios need to be carefully taken into consideration. In terms of the characterization of the dependence structure between variables, one of the most commonly used methods is the Copula model. Many scholars confirmed that the Copula model can be used to investigate the marginal distributions of variables and the dependence structure between variables regardless of the forms of their marginal distributions [1-4]. There are many different forms for the Copula model which possesses strong flexibility and expandability. Hence, it has a very wide scope of application. For example, based on the Pair-Copula model, Li and Cheng [5] proposed a setting model of futures' maintenance margins, which gives sufficient consideration to the risk hedge between futures contracts. In brief, the Copula model can well describe the dependence structure between futures contracts. However, Wang et al. [6] pointed that the Copula model fails to estimate the tail risk. As for effectively depicting the tail risk, the extreme value theory (EVT) shows an obvious superiority, because it directly focuses on the tail and thus can estimate and forecast the risk more accurately. A lot of previous works [7-12] reported that the peaks over threshold (POT) model of EVT is capable of fully using sample information on extreme values. There is a plentiful of literature in this field. For instance, Gilli and Këllezi [13] introduced the EVT to measure the financial risk. Based on the EVT, Liu and Wang [14] constructed a model to set the maintenance margins for a single futures contract, but they did not consider the setting of futures portfolios' maintenance margins. Additionally, the choice of the risk measure method is indispensable to accurately estimate the overall risk of futures portfolios, and hence it largely influences the effectiveness of the maintenance margins. The widely used risk measure is the Value at Risk (VaR), which does not conform to the consistency axiom and cannot reflect the investors' risk aversion. To overcome the drawbacks of VaR, Acerbi [15] put forward a new risk measure, namely, the power spectral risk measures (PSRM), and indicated that the PSRM has the features of the consistency and the risk aversion. Following Acerbi [15], there are a lot of developments; for details, see Cotter and Dowd [16], Dowd et al. [17], and Han et al. [18]. They came to a similar conclusion that the PSRM is superior to the VaR in terms of the subadditive property as well as the description of the tail information.

In a word, the existing studies on futures maintenance margins not only neglect that futures investors tend to hold futures portfolio and there exist a significant risk hedge and risk diversion between future contracts but also ignore the characteristics of the tail risk and the choice of the risk measure method. Therefore, it is obvious that we should not separately study the maintenance margins for a single futures contact. So the existing studies on setting maintenance margins for a single futures contact cannot satisfy the needs of practical operation in the futures market. That is why we explore the setting of the maintenance margins for the futures portfolio in our study. In order to cope with the above-listed problems, we develop a new method for setting futures portfolios' maintenance margins by combining three models, namely, the GARCH $(1,1)$ - $t$ model nested multivariate $t$-Copula, the peaks over threshold (POT) model, and the power spectral risk measures (PSRM). Moreover, considering that the trend of the percentage margins system is irreversible, in our method, the maintenance margins for each futures portfolio are stated as some percentages of the value of the futures portfolio.

The remainder of this paper is organized as follows. The next section shows the related methodologies. Section 3 encompasses the data set and the empirical results. In Section 4, we present the conclusions and implication.

\section{Methodology}

Assume that the number of futures contracts in a futures portfolio (denoted as pf) is $n . \omega_{i}(i=1,2, \ldots, n)$ represents the proportion of the position of futures contract $i$ in the gross position of the futures portfolio pf, and thus $\omega_{i}$ meets the condition: $\sum_{i=1}^{n} \omega_{i}=1$. Let $P_{i, t}$ denote the daily closing price of the futures contract $i$ on day $t$. The daily return of the futures contract $i$ on day $t$, that is, $R_{i, t}$, is defined as

$$
R_{i, t}=\ln \left(\frac{P_{i, t}}{P_{i, t-1}}\right) .
$$

Han et al. [18] considered the enforcement of the shortselling mechanism and separately studied the long position and the short position. In this study, we define the loss rates of the futures contract $i$ in terms of the long position and the short position on day $t$ as follows:

$$
\begin{aligned}
& L_{i, t}^{+}=1-\exp \left(R_{i, t}\right), \\
& L_{i, t}^{-}=\exp \left(R_{i, t}\right)-1 ;
\end{aligned}
$$

where $L_{i, t}^{+}$and $L_{i, t}^{-}$represent the loss rates of the long position and the short position of the futures contract $i$ on day $t$, respectively. Based on (1)-(2), the loss rate of the futures contract $i$ on day $t$ is described as

$$
L_{i, t}=(-1)^{\operatorname{sign}(x)}\left[\exp \left(R_{i, t}\right)-1\right]^{\operatorname{sign}^{\prime}(x)}
$$

here,

$$
\begin{aligned}
& \operatorname{sign}(x)= \begin{cases}0, & \text { if } x \text { is the long position } \\
1, & \text { if } x \text { is the short position, }\end{cases} \\
& \operatorname{sign}^{\prime}(x)= \begin{cases}+, & \text { if } x \text { is the long position } \\
-, & \text { if } x \text { is the short position. }\end{cases}
\end{aligned}
$$

On the basis of the portfolio theory, the return $R_{\mathrm{pf}, t}$ and the loss rate $L_{\mathrm{pf}, t}$ of the futures portfolio pf on day $t$ can be, respectively, defined as

$$
\begin{aligned}
& R_{\mathrm{pf}, t}=\sum_{i=1}^{n} \omega_{i} R_{i, t}, \\
& L_{\mathrm{pf}, t}=\sum_{i=1}^{n} \omega_{i} L_{i, t} .
\end{aligned}
$$


In this paper, we propose a new method, namely, the multivariate $t$-Copula-POT-PSRM method, which contains four steps to set the maintenance margins of the futures portfolio pf. Firstly, we employ the GARCH $(1,1)$ - $t$ model nested multivariate $t$-Copula to describe the covariance and the dependence structure. Subsequently, by combining the quantitative finance theory and portfolio theory, we measure the standardized residual and variance of the loss rate of the futures portfolio pf. Thirdly, we focus directly on the tail of the futures portfolio pf by modeling the extreme tail of the standardized residual using the POT model, and we also estimate the quantile of the standardized residual sequence under a certain significant level. Finally, the dynamic risk of the futures portfolio pf is estimated by PSRM, which is an important part of setting the maintenance margins.

2.1. Modeling Covariance and Dependence Structure of Futures Contracts. Based on the characteristics of the Copula model, we use a two-step process that includes the modeling of marginal distributions and the application of the Copula function, to isolate the dependence structure from the marginal distributions.

2.1.1. Modeling Marginal Distributions. Considering that the $\operatorname{GARCH}(1,1)-t$ model is superior in analyzing and forecasting the volatilities of the financial data, we select the GARCH $(1,1)-t$ model to estimate the marginal distributions. According to the study of Bollerslev [19], the GARCH $(1,1)-t$ model is showed as

$$
\begin{gathered}
L_{i, t}=u_{i}+\sigma_{i, t} z_{i, t}, \\
\sigma_{i, t}^{2}=c+\alpha \varepsilon_{i, t-1}^{2}+\gamma \sigma_{i, t-1}^{2}, \\
f(x, m)=\frac{\Gamma(m+1 / 2)}{(m \pi)^{1 / 2} \Gamma(m / 2)}\left[1+\left(x^{2} / m\right)\right]^{-(m+1) / 2},
\end{gathered}
$$

where $L_{i, t}$ and $\sigma_{i, t}$ are the conditional mean and the conditional standard deviation of the loss rate of the futures contract $i$, respectively; $\varepsilon_{i, t}$ refers to the disturbance term of the mean equation; $\left\{z_{i, t}\right\}$ obeys a $t$-distribution and its degree of freedom is $m ; \Gamma(\cdot)$ means the Gamma distribution function; and (8) is the probability density function of the $t$-distribution. In GARCH $(1,1)$ - $t$ model, the parameters are $u, c, \alpha, \gamma$, and $m$, which meet the constraint conditions $c>0$, $\alpha \geq 0, \gamma \geq 0$, and $\alpha+\gamma<1$.

2.1.2. Constructing Multivariate $t$-Copula Function. After confirming the marginal distributions of futures contracts, we model the dependence structure between futures contracts based on the multivariate $t$-Copula function which can be used to study the marginal distributions and the dependence structure. The multivariate $t$-Copula function defined by Nikoloulopoulos et al. [20] is showed as follows:

$$
\begin{aligned}
& \mathrm{C}\left(u_{1}, u_{2}, \ldots, u_{n} ; \rho, v\right) \\
& =\mathrm{T}_{\rho, v}\left(\mathrm{~T}_{v}^{-1}\left(u_{1}\right), \mathrm{T}_{v}^{-1}\left(u_{2}\right), \ldots, \mathrm{T}_{v}^{-1}\left(u_{n}\right)\right)
\end{aligned}
$$

$$
\begin{gathered}
=\int_{-\infty}^{\mathrm{T}_{v}^{-1}\left(u_{1}\right)} \int_{-\infty}^{\mathrm{T}_{v}^{-1}\left(u_{2}\right)} \int_{-\infty}^{\mathrm{T}_{v}^{-1}\left(u_{n}\right)} \cdots \frac{\Gamma((v+n) / 2)|\rho|^{-1 / 2}}{\Gamma(v / 2)(v \pi)^{n / 2}} \\
\times\left(1+\frac{1}{v} x^{\prime} \rho^{-1} x\right)^{-(v+n) / 2} \mathrm{~d} x_{1} \mathrm{~d} x_{2} \cdots \mathrm{d} x_{n}, \\
\mathrm{C}\left(u_{1}, u_{2}, \ldots, u_{n} ; \rho, v\right) \\
=\left|\rho^{-1 / 2}\right| \frac{\Gamma((v+n) / 2)[\Gamma(v / 2)]^{n-1}}{[\Gamma((v+1) / 2)]^{n}} \\
\quad \times \frac{\left(1+(1 / v) \varsigma^{\prime} \rho^{-1} \varsigma\right)^{-(v+n) / 2}}{\prod_{i=1}^{n}\left(1+\varsigma_{i}^{2} / v\right)^{-(v+1) / 2}}, \\
x=\left(x_{1}, x_{2}, \ldots, x_{n}\right)^{\prime}, \quad \varsigma=\left(\varsigma_{1}, \varsigma_{2}, \ldots, \varsigma_{n}\right)^{\prime}, \\
\varsigma_{i}=\mathrm{T}_{v}^{-1}\left(u_{i}\right), \quad i=1,2, \ldots, n,
\end{gathered}
$$

where $\rho$ is a symmetrical positive definite matrix and all of its diagonal elements are equal to 1 , and its determinant is denoted as $|\rho| ; \mathrm{T}_{\rho, v}(., \ldots,$.$) represents the distribution$ function of the standardized multivariate $t$-distribution with a correlation coefficient matrix $\rho$ and degrees of freedom $v$; $\mathrm{T}^{-1} v(\cdot)$ is the inverse distribution function of $t$-distribution with $v$ degrees of freedom.

2.2. Estimating the Variance of the Futures Portfolio pf. To set the maintenance margins of the futures portfolio pf, we need to figure out the variance of the futures portfolio pf. On the basis of the quantitative finance theory and portfolio theory, the mean and variance of the loss rate of the futures portfolio pf can be, respectively, expressed as

$$
\begin{gathered}
L_{\mathrm{pf}, t}=u_{\mathrm{pf}, t}+\varepsilon_{\mathrm{pf}, t}=u_{\mathrm{pf}, t}+\sigma_{\mathrm{pf}, t} Z_{\mathrm{pf}, t}=\sum_{i=1}^{n} \omega_{i} u_{i, t}+\sigma_{\mathrm{pf}, t} Z_{\mathrm{pf}, t}, \\
\sigma_{\mathrm{pf}, t}^{2}=\sum_{i=1}^{n} \sum_{j=1}^{n} \omega_{i} \omega_{j} \sigma_{i j, t}=\sum_{i=1}^{n} \sum_{j=1}^{n} \omega_{i} \omega_{j} \sigma_{i, t} \sigma_{j, t} \rho_{i j}
\end{gathered}
$$

where $u_{\mathrm{pf}, t}$ is the conditional mean of the loss rate of the futures portfolio pf on day $t$, and $\left\{Z_{\mathrm{pf}, t}\right\}$ means the standardized residual sequence of the loss rate of the futures portfolio pf; $u_{i, t}$ refers to the conditional mean of the loss rate of the futures contract $i$, while $\sigma_{i, t}$ and $\sigma_{j, t}$ are the standard deviations of the loss rates of futures contracts $i$ and $j$, respectively; and $\sigma_{i j, t}$ denotes the covariance of the loss rates of futures contracts $i$ and $j$.

Here, we deem that $\rho_{i j}$ in (13), which connotes the correlation coefficient between futures contracts $i$ and $j$, is decided by the correlation coefficient matrix $\rho$ in (10) and (11). Meanwhile, for futures contracts $i$ and $j$, the following conditions need to be met: $\sigma_{i j, t}=\sigma_{j i, t}, \rho_{i j}=\rho_{j i}, \rho_{i i}=\rho_{j j}=1$, and $\sigma_{i i, t}=\sigma_{i, t}^{2}, \sigma_{j j, t}=\sigma_{j, t}^{2}$. 
2.3. Depicting Tail Features of the Futures Portfolio pf. In order to set proper maintenance margins, we should emphasize on the tail risk of the futures portfolio pf, which is embodied in the standardized residual sequence $\left\{Z_{\mathrm{pf}, t}\right\}$. Smith [21] pointed out that the observations of $\left\{Z_{\mathrm{pf}, t}\right\}$ over a high value $\mu$ in the POT model are assumed to be independent and to obey arbitrary common distributions:

$$
\begin{aligned}
F_{\mu}(z) & =P\{Z-\mu \leq z \mid Z>\mu\}=\frac{P\{Z-\mu \leq z, Z>\mu\}}{P\{Z>\mu\}} \\
& =\frac{P\{\mu<Z \leq z+\mu\}}{P\{Z>\mu\}}=\frac{F(z+\mu)-F(\mu)}{1-F(\mu)},
\end{aligned}
$$

where $Z$ refers to a random variable, $z$ denotes the right endpoint of the range of $Z$, and $F$ is the distribution function of $Z$, while $F_{\mu}(z)$ represents the distribution function of all observations which exceed a given threshold $\mu$. All observations larger than $\mu$ (which is large enough) obey the generalized Pareto distribution (GPD).Based on the researches of Balkema and de Haan [22] and Pickands III [23], $F_{\mu}(z)$ can also be denoted as

$$
F_{\mu}(z)= \begin{cases}1-\exp \left(-\frac{z-\mu}{\beta}\right), & \xi=0, z \in\left[\mu,-\frac{\beta}{\xi}+\mu\right], \\ 1-\left(1+\xi \frac{z-\mu}{\beta}\right)^{-1 / \xi}, & \xi \neq 0, z \in[\mu,+\infty),\end{cases}
$$

where $\beta$ and $\xi$ are parameters. Obviously, the fitting effect of POT model, to a large degree, depends on the rationality of the value of $\mu$. To obtain the value of $\mu$, we adopt the mean excess plotting, which is the point set of $\{\mu, e(\mu)\}$ [24]. The expression of $e(\mu)$ is defined by

$$
\begin{array}{r}
e(\mu)=E(Z-\mu \mid Z>\mu)=\frac{1}{N^{*}} \sum_{i=1}^{N^{*}}(z(i)-\mu), \\
0 \leq \mu \leq z(i),
\end{array}
$$

where $z(i), i=1,2, \ldots, N^{*}$ refers to the observations of $\left\{Z_{\mathrm{pf}, t}\right\}$ and $N^{*}$ represents the number of observations exceeding $\mu$. An appropriate threshold $\mu$ should meet the following condition: the mean excess plotting presents a linear momentum, starting from the point where its abscissa value is $\mu$. Here, we can roughly use $\left(n-N^{*}\right) / n$ to estimate $F(\mu)$. Furthermore, based on (15) and (16), we can deduce the $q$ quantile of $\left\{Z_{\mathrm{pf}, t}\right\}$, that is,

$$
\begin{aligned}
F_{z}^{\leftarrow}(q) & =\inf \left\{z \mid F_{z}(z) \geq q\right\} \\
& =\mu+\frac{\beta}{\xi}\left\{\left[\frac{N}{N^{*}}(1-q)\right]^{-\xi}-1\right\} .
\end{aligned}
$$

2.4. Setting the Maintenance Margins of the Futures Portfolio pf. An appropriate risk measure plays an important role in estimating risk and setting maintenance margins. Acerbi [15] pointed that PSRM can not only reflect the risk aversion, but also perfectly fit the fact that the absolute risk aversion coefficient decreases progressively while the relative risk aversion coefficient roughly stays as a constant. Thereby, we employ PSRM, to estimate the risk of the future portfolio pf, and then set its maintenance margins. The PSRM is defined as

$$
\begin{array}{r}
\operatorname{PSRM}=\int_{0}^{1} X_{\alpha} \varphi(p)=\int_{0}^{1} X_{\alpha}(1-a)(1-p)^{-a} \mathrm{~d} p, \\
0<a<1,
\end{array}
$$

where $\varphi(\cdot)$ is a risk aversion function, $X_{\alpha}$ represents the $\alpha$ quantile, $a$ refers to the relative risk aversion coefficient, and $p$ stands for the cumulative probability. Dowd et al. [17] manifested that PSRM is sensitive to the changes of volatility when the relative risk aversion coefficient $a$ is equal to 0.7 . Hence, following Dowd et al. [17], we set $a$ as 0.7. By using PSRM, we estimate the overall risk of the futures portfolio pf. Combining (13), (17), and (18), the dynamic risk of the futures portfolio pf can be defines as

$$
\begin{aligned}
\operatorname{PSRM}_{t}=\int_{0}^{1}\left\{\sum_{i=1}^{n} \omega_{i} u_{i, t}+\sqrt{\sum_{i=1}^{n} \sum_{j=1}^{n} \omega_{i} \omega_{j} \sigma_{i, t} \sigma_{j, t} \rho_{i j}}\right. \\
\left.\times\left[\mu+\frac{\beta}{\xi}\left(\left(\frac{N}{N^{*}}(1-q)\right)^{-\xi}-1\right)\right]\right\} \\
\times\left[(1-a)(1-p)^{-a}\right] \mathrm{d} p .
\end{aligned}
$$

Subsequently, we can obtain the computational formula of Margin $_{t}$, which means the maintenance margin of the futures portfolio of on day $t$, that is,

$$
\operatorname{Margin}_{t}=\left\lceil\left|\operatorname{PSRM}_{t}\right|\right\rceil
$$

where $\lceil x\rceil$ stands for the smallest integer not smaller than $x$.

\section{Data and Empirical Results}

3.1. Data Set and Statistical Analysis. Based on the differences in the varieties and the trading locations of the popular futures contracts in the Chinese commodity futures markets, we construct four kinds of futures portfolios, denoted as Futures Portfolio A, Futures Portfolio B, Futures Portfolio C, and Futures Portfolio D. Futures Portfolio A involves the Number 1 Soybeans futures contract from DCE, the Copper Cathode futures contract from SHFE, and the Cotton futures contract from ZCE. Futures Portfolio B encompasses the Number 1 Soybeans futures contract, the Copper Cathode futures contract, and the Crude Soybean Oil futures contract from DCE. Futures Portfolio C includes the Copper Cathode futures contract, the Cotton futures contract, and the Crude Soybean Oil futures contract. Futures Portfolio D is made of the Number 1 Soybeans futures contract, the Cotton futures contract, and the Crude Soybean Oil futures contract. 
The distribution of the gross position along with the directions of futures positions for all four kinds of Futures Portfolios are shown in Table 1. In Table 1, taking Futures Portfolio A for example, 4/9, 3/9, and 2/9 refer to the proportions of the long Number 1 Soybeans position, the short Copper Cathode position, and the long Cotton position in the gross position. The data set consists of the daily closing prices of the nearby contracts of the Number 1 Soybeans, the Copper Cathode, the Cotton and the Crude Soybean Oil futures contracts from January 4, 2007 to June 28, 2013. We choose the period from January 4, 2007 to December 31, 2012 as the In-sample period to estimate the parameters of our method and set the period from January 4, 2013 to June 28, 2013 as the Out-of-sample period to test the effectiveness of our method. We obtain the empirical data from the websites of the Dalian Commodity Exchange (http://www.dce.com.cn), the Shanghai Commodity Exchange (http://www.shfe.com.cn), and the Zhengzhou Commodity Exchange (http://www.czce.com.cn).

Table 2 presents a series of statistics and test results for the return series of four futures contracts and their portfolios, that is, Futures Portfolio A, Futures Portfolio B, Futures Portfolio C, and Futures Portfolio D. As revealed in Table 2, for the return series of each portfolio, its mean and standard deviation are smaller than the smallest return series and bigger than the biggest one, which illustrates the existence of risk hedge and risk diversification. In Table 2, one can find that all the values of kurtosis and skewness are greater than 3 and not equal to 0 , respectively, which indicates that all the return series are fat-tailed. The fat-tailed phenomenon also can be confirmed by the Jarque-Bera statistics that reject the null hypothesis of Gaussian distribution at the $1 \%$ significance level. For each sequence, the ARCH(1)-LM statistic rejects the null hypothesis at the $1 \%$ significance level, which implies that the heteroscedasticity exists in the return series. The results of ADF test demonstrate that all the eight return series are stationary series at the $1 \%$ significance level.

3.2. Estimation of Parameters. The estimated parameters in our method mainly include the parameters of marginal distribution models, the degree of freedom of multivariate $t$-Copulas function, the correlation coefficient matrix, and the parameters of the POT model. In Table 3 , the estimates of $\alpha$ and $\gamma$ are positive, which proves the existence of the volatility clustering phenomenon in all the four series. For each sequence, the degree of freedom $m$ is significant, which confirms that all series are fat-tailed. Moreover, the values of $\alpha+\gamma$ are close to 1 , which implies that each series has strong persistence in volatility.

We employ the two-stage maximum likelihood estimation method to estimate the parameters of the multivariate $t$ Copula function. To be more specific, the degree of freedom of the multivariate $t$-Copula model based on the Number 1 Soybeans, Copper Cathode, and Cotton futures contracts is equal to 2.5318, the degree of freedom of the multivariate $t$ Copula model on basis of the Number 1 Soybeans, Copper Cathode, and Cotton futures contracts is equivalent to 3.267, the degree of freedom of the multivariate $t$-Copula function based on the Copper Cathode, Cotton, and Crude Soybean

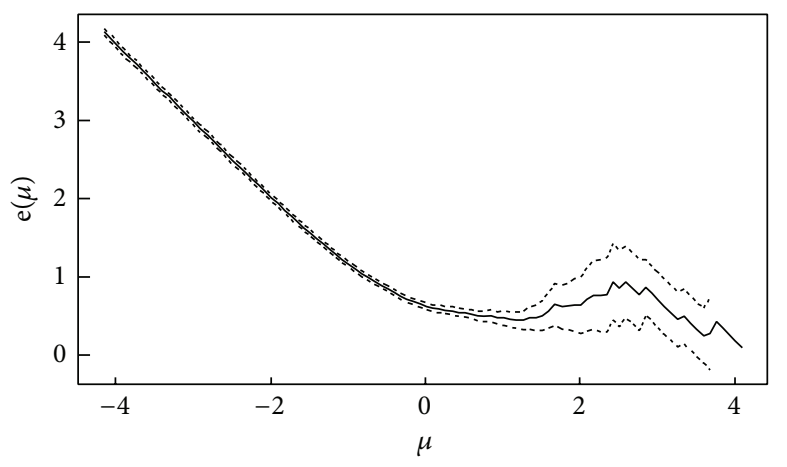

FIGURE 1: Mean excess plotting for the standardized residual sequence of the loss rate of the Futures Portfolio A. The solid line is the mean excess plotting. The region between the two dotted lines refers to the $95 \%$ confidence interval.

Oil futures contracts is equal to 3.1253 , and that of the multivariate $t$-Copula function on basis of the Number 1 Soybeans, Cotton, and Crude Soybean Oil futures contracts is equivalent to 2.643. Moreover, we list the correlation coefficient matrix in Table 4 . Table 4 illustrates that there exists the low positive or negative correlation among the three series, which verifies that the portfolio investment strategy is advisable on this occasion.

Considering the length of the paper, we take Future Portfolio A as an example to describe the detailed process of the estimation parameters of the POT model. We determine the value of $\mu$ by employing the mean excess plotting and show the graph in Figure 1. As drawn in Figure 1, the solid curve shows an almost linear trend when $\mu$ is in the interval $(0,2)$. Therefore, we initially identify $\mu$ in the interval $(0$, 2). To accurately position $\mu$ further, we present the fitting effect graph of the POT model with $\mu$ in the interval $(0,2)$ in Figure 2. When $\mu$ is equal to 1.1000 , as shown in Figure 2, the values of $\beta$ and $\xi$ roughly level off, which implies that 1.1000 is an appropriate value. In such case, the corresponding value of $N^{*}$ is 117. Then, we use the maximum likelihood method to estimate the parameters of the POT model, and the loglikelihood function is denoted as follows:

$$
\mathrm{L}(\xi, \beta \mid z)= \begin{cases}-n \ln \beta-\left(1+\frac{1}{\xi}\right) \sum_{k=1}^{n} \ln \left(1+\frac{\xi}{\beta} z_{k}\right), & \xi \neq 0, \\ -n \ln \beta-\frac{1}{\beta} \sum_{k=1}^{n} z_{k}, & \xi=0 .\end{cases}
$$

Finally, we calculate the values of $\beta$ and $\xi$ for Future Portfolio A as 0.4089 and 0.1102 , respectively. The value of the shape parameter $\xi$ is positive, which indicates that the loss rate of Futures Portfolio A is fat-tailed. Similarly, we obtain the results for the other three futures portfolios as follows: the values of $\beta$ and $\xi$ for Futures Portfolio B are equal to 0.3872 and 0.0965 ; the values of $\beta$ and $\xi$ for Futures Portfolio $C$ are equivalent to 0.4152 and 0.0731 ; and the values of $\beta$ and $\xi$ for Futures Portfolio D are equal to 0.3167 and 0.0638 .

After determining the parameters of our method, we can calculate the PSRM values based on (18). In 1995, reference 
TABLE 1: Allocation of the gross position for four kinds of Futures Portfolios.

\begin{tabular}{|c|c|c|c|c|}
\hline Futures Portfolio & Futures contracts & Commodity futures exchange & Long position & Short position \\
\hline \multirow{3}{*}{ Portfolio A } & Number 1 Soybeans & DCE & $4 / 9$ & 0 \\
\hline & Copper Cathode & SHFE & 0 & $3 / 9$ \\
\hline & Cotton & ZCE & $2 / 9$ & 0 \\
\hline \multirow{3}{*}{ Portfolio B } & Number 1 Soybeans & DCE & $4 / 9$ & 0 \\
\hline & Copper Cathode & SHFE & 0 & $3 / 9$ \\
\hline & Crude Soybean Oil & DCE & $2 / 9$ & 0 \\
\hline \multirow{3}{*}{ Portfolio C } & Copper Cathode & SHFE & $4 / 9$ & 0 \\
\hline & Cotton & $\mathrm{ZCE}$ & 0 & $3 / 9$ \\
\hline & Crude Soybean Oil & DCE & $2 / 9$ & 0 \\
\hline \multirow{3}{*}{ Portfolio D } & Number 1 Soybeans & DCE & $4 / 9$ & 0 \\
\hline & Cotton & $\mathrm{ZCE}$ & 0 & $3 / 9$ \\
\hline & Crude Soybean Oil & $\mathrm{DCE}$ & $2 / 9$ & 0 \\
\hline
\end{tabular}

TABLE 2: Descriptive statistics of return series.

\begin{tabular}{lcccccccc}
\hline Parameters & $\begin{array}{c}\text { Number } 1 \\
\text { soybeans }\end{array}$ & $\begin{array}{c}\text { Copper } \\
\text { cathode }\end{array}$ & Cotton & $\begin{array}{c}\text { Crude } \\
\text { soybean oil }\end{array}$ & Portfolio A & Portfolio B & Portfolio C & Portfolio D \\
\hline Mean & 0.0004 & 0.0003 & -0.0001 & 0.0002 & 0.0002 & 0.0003 & 0.0001 & 0.0002 \\
Maximum & 0.0612 & 0.0791 & 0.1079 & 0.0768 & 0.0377 & 0.0738 & 0.0437 & 0.0519 \\
Minimum & -0.0583 & -0.1061 & -0.1343 & -0.1316 & -0.0449 & -0.0664 & -0.0915 & -0.0827 \\
Std-Dev & 0.0115 & 0.0184 & 0.0121 & 0.0162 & 0.0093 & 0.0103 & 0.0095 & 0.0118 \\
Kurtosis & 8.8896 & 5.4383 & 4.0254 & 8.3548 & 5.8681 & 6.1527 & 5.8674 & 6.4317 \\
Skewness & -0.1825 & -0.4028 & -0.2069 & -0.8634 & -0.4438 & -0.5167 & -0.6872 & -0.7105 \\
& 1976.0640 & 1746.2950 & 2510.1400 & 1435.2574 & 511.5199 & 1176.0864 & 987.1367 & 1039.5378 \\
Jarque-Bera & $(0.0000)$ & $(0.0000)$ & $(0.0000)$ & $(0.0000)$ & $(0.0000)$ & $(0.0000)$ & $(0.0000)$ & $(0.0000)$ \\
& 123.9881 & 44.7033 & 31.8447 & 36.2749 & 72.3283 & 83.1259 & 46.8973 & 93.6217 \\
ARCH(1)-LM & $(0.0000)$ & $(0.0009)$ & $(0.0000)$ & $(0.0000)$ & $(0.0000)$ & $(0.0000)$ & $(0.0000)$ & $(0.0000)$ \\
& -18.8755 & -19.3994 & -22.0336 & -21.7358 & -19.0045 & -19.8639 & -20.1693 & -19.9476 \\
ADF & $(0.0000)$ & $(0.0000)$ & $(0.0000)$ & $(0.0000)$ & $(0.0000)$ & $(0.0000)$ & $(0.0000)$ & $(0.0000)$ \\
\hline
\end{tabular}

Notes: the figures in parenthesis denote $P$ values of statistics. Std-Dev stands for the standard deviation. The Jarque-Bera statistic tests for the null hypothesis of normality distribution. The ARCH(1)-LM statistic tests for the null hypothesis of the inexistence of heteroscedasticity until the lag order is equal to 1 . For the ADF test, the number of lags is estimated through the Akaike Information Criterion (AIC).

TABLE 3: Estimation of parameters of marginal distribution models.

\begin{tabular}{lcccc}
\hline Parameters & Number 1 soybeans & Copper cathode & Cotton & Crude soybean oil \\
\hline$u$ & $-0.0003^{*}(-1.7553)$ & $0.0006^{*}(1.7566)$ & $-0.0001(-0.7287)$ & $0.0004(-0.9183)$ \\
$c$ & $1.3556 e-5^{* * *}(2.9357)$ & $5.6875 e-6^{* * *}(2.4469)$ & $2.9713 e-6^{* * *}(3.4973)$ & $3.5372 e-6^{* * *}(3.5842)$ \\
$\alpha$ & $0.2714^{* * *}(3.2369)$ & $0.1146^{* * *}(5.0954)$ & $0.1698^{* * *}(4.7560)$ & $0.1064^{* * *}(4.8432)$ \\
$\gamma$ & $0.7286^{* * *}(16.5762)$ & $0.8775^{* * *}(42.5026)$ & $0.8302^{* * *}(37.4746)$ & $0.8637^{* * *}(26.8547)$ \\
$m$ & $2.7196^{* * *}(10.1095)$ & $4.9958^{* * *}(6.8156)$ & $3.0547^{* * *}(10.6815)$ & $5.0262^{* * *}(8.8953)$ \\
\hline
\end{tabular}

Notes: the figures in parenthesis denote the value of $t$ statistics. The asterisks ${ }^{* * *},{ }^{* *}$, and ${ }^{*}$ represent the significance level at the $1 \%, 5 \%$, and $10 \%$, respectively.

TABLE 4: Correlation coefficient matrix of futures contracts.

\begin{tabular}{|c|c|c|c|c|}
\hline & Number 1 soybeans & Copper cathode & Cotton & Crude soybean oil \\
\hline Number 1 soybeans & 1.0000 & -0.2493 & 0.0028 & 0.0014 \\
\hline Copper cathode & -0.2493 & 1.0000 & -0.0076 & -0.0032 \\
\hline Cotton & 0.0028 & -0.0076 & 1.0000 & 0.0062 \\
\hline Crude soybean oil & 0.0014 & -0.0032 & 0.0062 & 1.0000 \\
\hline
\end{tabular}


TABLE 5: The results of the Kupiec test for the PSRM method.

\begin{tabular}{lcccc}
\hline & & The number of the failure days & The failure rate & LR statistics \\
\hline \multirow{3}{*}{ Portfolio A } & $P^{*}=5 \%$ & 59 & 0.0502 & $0.0007(0.9787)$ \\
& $P^{*}=2.5 \%$ & 26 & 0.0221 & $0.4194(0.5173)$ \\
& $P^{*}=1 \%$ & 9 & 0.077 & $0.7119(0.3988)$ \\
\hline \multirow{3}{*}{ Portfolio B } & $P^{*}=5 \%$ & 47 & 0.0400 & $2.6477(0.1037)$ \\
& $P^{*}=2.5 \%$ & 17 & 0.0145 & $6.2875(0.0122)$ \\
& $P^{*}=1 \%$ & 5 & 0.0043 & $4.9949(0.0254)$ \\
\hline \multirow{3}{*}{ Portfolio C } & $P^{*}=5 \%$ & 54 & 0.0459 & $0.4150(0.5195)$ \\
& $P^{*}=2.5 \%$ & 26 & 0.0060 & $0.4134(0.5202)$ \\
Portfolio D & $P^{*}=1 \%$ & 7 & 0.0545 & $2.2682(0.1321)$ \\
\hline$P^{*}=5 \%$ & 64 & 0.0323 & $0.4804(0.4882)$ \\
& $P^{*}=2.5 \%$ & 38 & 0.0102 & $2.3807(0.1228)$ \\
\hline
\end{tabular}

Notes: the figures in parenthesis denote the $P$ value of the LR statistics.
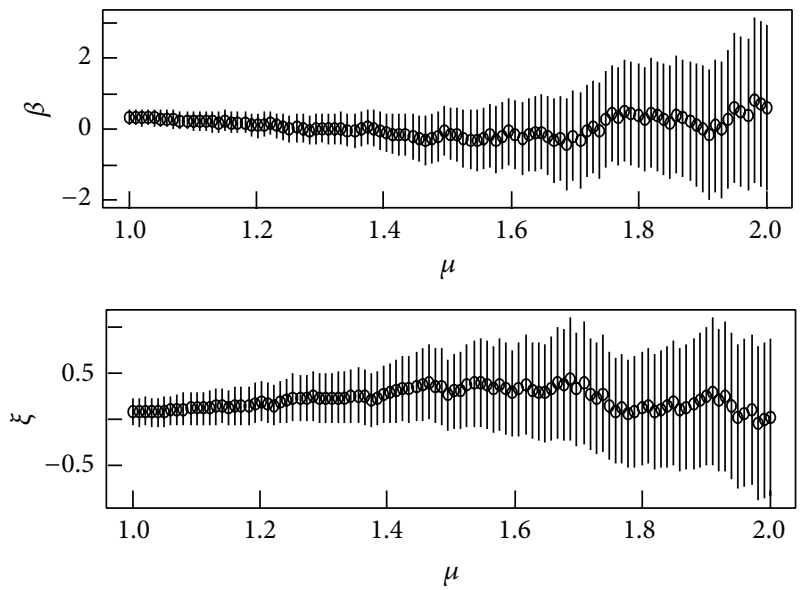

FIGURE 2: Fitting effect graph of the POT model with $\mu$ in the interval $(0,2)$ for the Futures Portfolio A.

[25] proposed the Kupiec test which is used to quantify the accuracy of an institution's VaR estimates. Thus, following [25], we use the Kupiec test to verify the accuracy of the PSRM method. The detailed procedures of the Kupiec test are represented as follows.

Firstly, we calculate the number of the failure days as well as the failure rate:

$$
\begin{gathered}
I_{t+1}= \begin{cases}1, & L_{\mathrm{pf}, t+1}<\operatorname{PSRM}_{t+1, t}, \\
0, & L_{\mathrm{pf}, t+1} \geq \operatorname{PSRM}_{t+1, t},\end{cases} \\
N=\sum_{t=1}^{T} I_{t+1}, \quad P_{\text {fail }}=\frac{N}{T},
\end{gathered}
$$

where $L_{\mathrm{pf}, t+1}$ stands for the actual loss rate of futures portfolio on day $t+1, P_{\text {fail }}$ is the failure rate, $\operatorname{PSRM}_{t+1, t}$ is the estimated value by using the PSRM method, and $T$ represents the length of the observation period. by

Then, based on Kupiec's work, the LR statistics is defined

$$
\begin{aligned}
\mathrm{LR}= & -2 \ln \left[\left(1-p^{*}\right)^{T-N}\left(p^{*}\right)^{N}\right] \\
& +2 \ln \left[\left(1-P_{\text {fail }}\right)^{T-N}\left(P_{\text {fail }}\right)^{N}\right],
\end{aligned}
$$

where $p^{*}$ is a given significance level.

According to the studies of Kupiec [25], if the null hypothesis, that is, $P_{\text {fail }}=p^{*}$, is met, the LR statistics should obey the Chi-square distribution with 1 degree of freedom. The specific rules of the Kupiec test are presented as follows. On the one hand, if the $p$ value of the LR statistics is smaller than $p^{*}$, we reject the null hypothesis. In such cases, when $P_{\text {fail }}$ is smaller than $p^{*}$, the PSRM method is too conservative so that it overestimates the risk, while when $P_{\text {fail }}$ is bigger than $p^{*}$, the PSRM method tends to underestimate the risk. On the other hand, if the $p$ value of the LR statistics is bigger than $p^{*}$ and $P_{\text {fail }}$ is close to $p^{*}$, we accept the null hypothesis, which implies that the PSRM method is effective in measuring and estimating the risk. We display the results of the Kupiec test in Table 5. From Table 5, we can find that the $p$ value of the LR statistics is greater than the corresponding significance level, and at the same time the failure rate $P_{\text {fail }}$ is close to $p^{*}$, which indicates that the PSRM can effectively measure and estimate the risk.

\subsection{Effectiveness Evaluation}

3.3.1. Evaluation Indicators. In order to accurately appraise the effectiveness of the proposed method, we introduce two indicators as proposed in [26], namely, the prudence index (PI) and the opportunity cost index (OCI). PI is defined as the probability at which the loss is not more than the maintenance margins. OCI connotes the average of overcharged 
maintenance margins, which reflects the opportunity cost of investors. The two indicators are defined as

$$
\begin{gathered}
\mathrm{PI}=\frac{1}{n} \sum_{t=1}^{n} I\left(\operatorname{Margin}_{A, t}>\left|L_{A, t}\right|\right), \\
\mathrm{OCI}=\frac{\sum_{t=1}^{n}\left(\operatorname{Margin}_{A, t}-\left|L_{A, t}\right|\right) I\left(\operatorname{Margin}_{A, t}>\left|L_{A, t}\right|\right)}{I\left(\operatorname{Margin}_{A, t}>\left|L_{A, t}\right|\right)},
\end{gathered}
$$

where $I(\cdot)$ is an indicative function and $M_{t}$ and $L_{t}$ represent the maintenance margins and the loss rate of the Futures Portfolio A on day $t$, respectively. More details about the two evaluation indicators (PI and OCI) can be seen in [26].

Considering that the main function of futures maintenance margins is to safeguard the safety and stabilities of the futures market, the sound and effective maintenance margins should firstly satisfy the security requirement and then try to reduce the transaction cost and improve the capital usage effectiveness. Hence, when we evaluate the effectiveness of the maintenance margins, we must abide by the following rules: (i) PI is the key indicator, and it should be seen as the first evaluation standard. Specifically, the larger the PI value is, the better the effectiveness of the maintenance margins is; (ii) when there exists no significant difference in the PI value, the OCI should be regarded as the evaluation standard. To be more exact, the smaller the OCI value is, the better the effectiveness of the maintenance margins is.

3.3.2. Effectiveness of the Proposed Method. When the relative risk aversion coefficient $a$ falls in the interval [0.5, 0.7], the results of the effectiveness of the maintenance margins for Futures Portfolios A, B, C, and D by using our method for the In-sample period as well as the Out-of-sample period are displayed in Table 6. From Table 6, we can find that the values of PI and OCI vary with different relative risk aversion coefficient $a$, which implies that the maintenance margins that are set by using our method can accordingly adjust to the change of the degree of risk aversion. That is to say, our method presents a relatively strong adaptability. Moreover, according to the rules of evaluating the effectiveness of the maintenance margins, we can find that when the relative risk aversion coefficient $a$ is equal to 0.7 , the effectiveness of the maintenance margins is the best for both the In-sample period and the Out-of-sample period. So we set the relative risk aversion coefficient $a$ to be 0.7 in our method.

\subsubsection{Comparison between Different Methods of Setting} the Maintenance Margins. In fact, the Chinese commodity futures exchanges implement the fixed futures margin system, and only on special occasions, such as legal holidays, they adjust maintenance margins. When the Chinese commodity futures exchanges charge futures portfolios' maintenance margins, they use a linear additivity model and ignore the risk diversification and hedge between futures contracts. For each futures contract, its maintenance margins vary in a certain fixed interval, and the interval is determined by the commodity futures exchanges without full consideration of the actual volatility of the futures contract.
TABLE 6: Effectiveness of our method under different relative risk

\begin{tabular}{|c|c|c|c|c|}
\hline & Interval & $a$ & PI & OCI \\
\hline \multirow{10}{*}{ Portfolio A } & \multirow{5}{*}{ In-sample } & 0.5 & 0.9674 & 0.0627 \\
\hline & & 0.6 & 0.9832 & 0.0671 \\
\hline & & 0.7 & 1.0000 & 0.0778 \\
\hline & & 0.8 & 1.0000 & 0.0815 \\
\hline & & 0.9 & 1.0000 & 0.0860 \\
\hline & \multirow{5}{*}{ Out-of-sample } & 0.5 & 0.9476 & 0.0501 \\
\hline & & 0.6 & 0.9657 & 0.0615 \\
\hline & & 0.7 & 0.9983 & 0.0709 \\
\hline & & 0.8 & 0.9994 & 0.0810 \\
\hline & & 0.9 & 1.0000 & 0.0852 \\
\hline \multirow{10}{*}{ Portfolio B } & \multirow{5}{*}{ In-sample } & 0.5 & 0.9356 & 0.0684 \\
\hline & & 0.6 & 0.9474 & 0.0575 \\
\hline & & 0.7 & 0.9792 & 0.06844 \\
\hline & & 0.8 & 1.0000 & 0.0858 \\
\hline & & 0.9 & 1.0000 & 0.0893 \\
\hline & \multirow{5}{*}{ Out-of-sample } & 0.5 & 0.9364 & 0.0674 \\
\hline & & 0.6 & 0.9582 & 0.0797 \\
\hline & & 0.7 & 0.9993 & 0.0847 \\
\hline & & 0.8 & 0.9995 & 0.0889 \\
\hline & & 0.9 & 1.0000 & 0.0904 \\
\hline \multirow{10}{*}{ Portfolio C } & \multirow{5}{*}{ In-sample } & 0.5 & 0.9467 & 0.0649 \\
\hline & & 0.6 & 0.9770 & 0.0666 \\
\hline & & 0.7 & 0.9867 & 0.0748 \\
\hline & & 0.8 & 1.0000 & 0.0806 \\
\hline & & 0.9 & 1.0000 & 0.0835 \\
\hline & \multirow{5}{*}{ Out-of-sample } & 0.5 & 0.9476 & 0.0501 \\
\hline & & 0.6 & 0.9657 & 0.0615 \\
\hline & & 0.7 & 0.9845 & 0.0694 \\
\hline & & 0.8 & 0.9906 & 0.0795 \\
\hline & & 0.9 & 1.0000 & 0.0847 \\
\hline \multirow{10}{*}{ Portfolio D } & \multirow{5}{*}{ In-sample } & 0.5 & 0.9575 & 0.0585 \\
\hline & & 0.6 & 0.9807 & 0.0697 \\
\hline & & 0.7 & 0.9897 & 0.0745 \\
\hline & & 0.8 & 1.0000 & 0.0873 \\
\hline & & 0.9 & 1.0000 & 0.0906 \\
\hline & \multirow{5}{*}{ Out-of-sample } & 0.5 & 0.9578 & 0.0567 \\
\hline & & 0.6 & 0.9667 & 0.0675 \\
\hline & & 0.7 & 0.9975 & 0.0693 \\
\hline & & 0.8 & 0.9981 & 0.0795 \\
\hline & & 0.9 & 1.0000 & 0.0814 \\
\hline
\end{tabular}
aversion coefficients.

Notes: $a$ stands for the relative risk aversion coefficient.

Taking Futures Portfolio A as an example, we compare the maintenance margins between our method (when the relative risk aversion coefficient $a$ is equal to 0.7) and the current linear additive model, and present the results for the Insample period in Figure 3. Obviously, the two kinds of maintenance margins can perfectly cover the futures portfolio's extreme risk, which suggests that they are capable of keeping risk within an acceptable range. However, compared with 


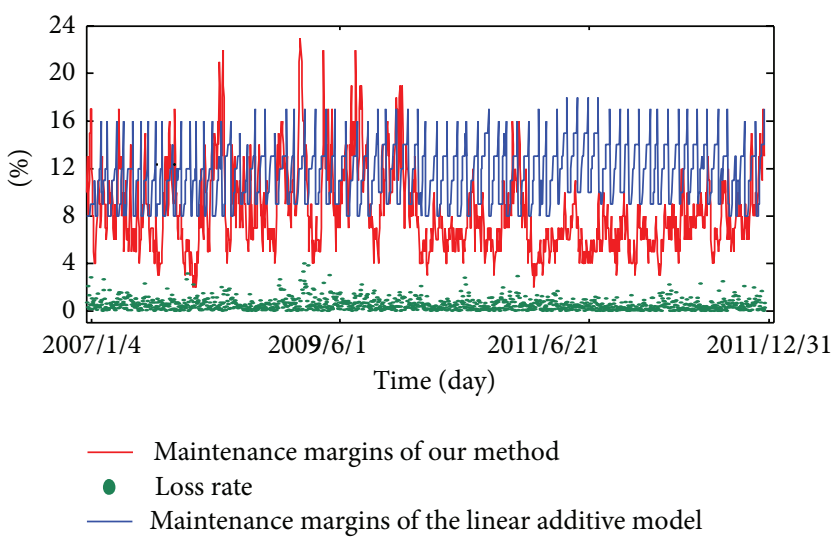

FIgURE 3: Comparison diagram of maintenance margins for Futures Portfolio A.

TABLE 7: Effectiveness of different methods of setting futures maintenance margins.

\begin{tabular}{|c|c|c|c|c|}
\hline & Interval & Model & PI & OCI \\
\hline \multirow{4}{*}{ Portfolio A } & \multirow{2}{*}{ In-sample } & Linear additive model & 1.0000 & 0.1080 \\
\hline & & Our new method & 1.0000 & 0.0778 \\
\hline & \multirow{2}{*}{ Out-of-sample } & Linear additive model & 0.9997 & 0.0978 \\
\hline & & Our new method & 0.9983 & 0.0709 \\
\hline \multirow{4}{*}{ Portfolio B } & \multirow{2}{*}{ In-sample } & Linear additive model & 1.0000 & 0.1036 \\
\hline & & Our new method & 1.0000 & 0.0745 \\
\hline & \multirow{2}{*}{ Out-of-sample } & Linear additive model & 0.9986 & 0.0964 \\
\hline & & Our new method & 0.9979 & 0.0694 \\
\hline \multirow{4}{*}{ Portfolio C } & \multirow{2}{*}{ In-sample } & Linear additive model & 0.9997 & 0.1046 \\
\hline & & Our new method & 0.9995 & 0.0815 \\
\hline & \multirow{2}{*}{ Out-of-sample } & Linear additive model & 0.9963 & 0.0848 \\
\hline & & Our new method & 0.9957 & 0.0615 \\
\hline \multirow{4}{*}{ Portfolio D } & \multirow{2}{*}{ In-sample } & Linear additive model & 1.0000 & 0.1080 \\
\hline & & Our new method & 1.0000 & 0.0778 \\
\hline & \multirow{2}{*}{ Out-of-sample } & Linear additive model & 0.9983 & 0.0815 \\
\hline & & Our new method & 0.9979 & 0.0695 \\
\hline
\end{tabular}

the linear additive model, there exist more significant ups and downs in the maintenance margins of Futures Portfolio A which are obtained by means of our method. This finding implies that our method is more flexible than the current approach.

In order to test the effectiveness, we compare our method with the current widely-used linear additive model for Futures Portfolios A, B, C, and D in terms of the values of PI as well as OCI for both the In-sample period and the Outof-sample period and display the results of the comparison in Table 7. When it comes to Futures Portfolio A, from Table 7 we can conclude that in terms of PI the newly proposed method shows a similar performance as the current linear additive model for both the In-sample period and the Out-ofsample period, while the value of OCI, respectively, decreases by $28 \%$ and $27.5 \%$ for the In-sample period and the Out-ofsample period after adopting the new method. Similarly, as for the other three futures portfolios, our new method shows superiority over the linear additive model with regard to the PI and OCI values for the In-sample period and the Outof-sample period. In short, our method can not only effectively cover the risk of futures portfolio, but also significantly reduce the opportunity cost of futures' investors and thus improve the capital investment efficiency.

\section{Conclusion}

In view of the problems in setting futures portfolios' maintenance margins for the Chinese commodity futures exchanges, we propose a new method that integrates the GARCH $(1,1)-t$ model nested multivariate $t$-Copula, the POT model, and PSRM. To verify the validity of the method, we firstly construct four kinds of futures portfolios, that is, the Future Portfolios A, B, C, and D, and then by using the new method set the maintenance margins of the Future Portfolios A, B, C, and D. Subsequently, for both the In-sample period 
and the Out-of-sample period, we assess the effectiveness of the proposed method, and compare the new method with the commonly used linear additive model with respect to the two evaluation indicators, namely, PI and OCI. The empirical results show that our new method can, respectively, lower the OCI value for the In-sample period and the Out-of-sample period for all four kinds of futures portfolios, which means that the occupied funds of futures portfolio holders can be largely released with the result of increased capital investment efficiency. Moreover, we find that the new method shows more flexibility compared to the current model, because the new method can better capture and cover the futures portfolio's risk, and it is also able to adjust the maintenance margins in accordance with the size and the direction of futures position.

\section{Conflict of Interests}

The authors declare that there is no conflict of interests regarding the publication of this paper.

\section{Acknowledgments}

The authors are grateful to the Editor and three anonymous reviewers for their valuable suggestions that significantly improved the quality of the paper. Gang-Jin Wang thanks the partial support from the Scholarship Award for Excellent Doctoral Student granted by the Ministry of Education of China, the Fundamental Research Funds for the Central Universities of Hunan University and the Hunan Provincial Innovation Foundation for Postgraduate (Grant no. CX2013A006). This work was supported by the National Natural Science Foundation of China (Grant no. 71373072), the Specialized Research Fund for the Doctoral Program of Higher Education (Grant no. 20130161110031) and the Foundation for Innovative Research Groups of the National Natural Science Foundation of China (Grant no. 71221001).

\section{References}

[1] G. Frahm, M. Junker, and R. Schmidt, "Estimating the taildependence coefficient: properties and pitfalls," Insurance, vol. 37, no. 1, pp. 80-100, 2005.

[2] E. I. George and S. T. Jensen, "A latent variable perspective of copula modeling," Marketing Science, vol. 30, no. 1, pp. 22-24, 2011.

[3] S. Kusuoka and T. Nakashima, "A remark on credit risk models and copula," Advances in Mathematical Economics, vol. 16, pp. 53-84, 2012.

[4] A. Panagiotelis, C. Czado, and H. Joe, "Pair copula constructions for multivariate discrete data," Journal of the American Statistical Association, vol. 107, no. 499, pp. 1063-1072, 2012.

[5] N. Li and X. J. Cheng, "A new method for setting dynamic futures portfolio margin level," Journal of University of Science and Technology of China, vol. 42, no. 3, pp. 198-202, 2012 (Chinese).

[6] Z.-R. Wang, X.-H. Chen, Y.-B. Jin, and Y.-J. Zhou, "Estimating risk of foreign exchange portfolio: using VaR and CVaR based on GARCHEVT-Copula model," Physica A, vol. 389, no. 21, pp. 4918-4928, 2010.

[7] F. J. Acero, J. A. García, and M. C. Gallego, "Peaks-overthreshold study of trends in extreme rainfall over the Iberian Peninsula," Journal of Climate, vol. 24, no. 4, pp. 1089-1105, 2011.

[8] J. Beirlant, E. Joossens, and J. Segers, "Second-order refined peaks-over-threshold modelling for heavy-tailed distributions," Journal of Statistical Planning and Inference, vol. 139, no. 8, pp. 2800-2815, 2009.

[9] V. Chavez-Demoulin and A. C. Davison, "Modelling time series extremes," Revstat Statistical Journal, vol. 10, no. 1, pp. 109-133, 2012.

[10] E. F. Eastoe and J. A. Tawn, "Modelling the distribution of the cluster maxima of exceedances of subasymptotic thresholds," Biometrika, vol. 99, no. 1, pp. 43-55, 2012.

[11] J. A. Chan-Lau, S. Mitra, and L. L. Ong, "Identifying contagion risk in the international banking system: an extreme value theory approach," International Journal of Finance and Economics, vol. 17, no. 4, pp. 390-406, 2012.

[12] I. Papastathopoulos and J. A. Tawn, "Extended generalised Pareto models for tail estimation," Journal of Statistical Planning and Inference, vol. 143, no. 1, pp. 131-143, 2013.

[13] M. Gilli and E. Këllezi, "An application of extreme value theory for measuring financial risk," Computational Economics, vol. 27, no. 2-3, pp. 207-228, 2006.

[14] Q. F. Liu and Z. Wang, "Setting dynamic margin levels in Chinese futures markets," Journal of Systems Engineering, vol. 26, no. 6, pp. 777-784, 2011 (Chinese).

[15] C. Acerbi, "Spectral measures of risk: a coherent representation of subjective risk aversion," Journal of Banking and Finance, vol. 26, no. 7, pp. 1505-1518, 2002.

[16] J. Cotter and K. Dowd, "Extreme spectral risk measures: an application to futures clearinghouse margin requirements," Journal of Banking and Finance, vol. 30, no. 12, pp. 3469-3485, 2006.

[17] K. Dowd, J. Cotter, and G. Sorwar, "Spectral risk measures: properties and limitations," Journal of Financial Services Research, vol. 34, no. 1, pp. 61-75, 2008.

[18] D. Z. Han, X. F. Wang, F. Xing, M. M. Yang, and Y. J. Lou, "Setting up of dynamic margin of futures based on spectral risk measurement of extreme value," Journal of Management Sciences, vol. 22, no. 1, pp. 86-94, 2009 (Chinese).

[19] T. Bollerslev, "A conditionally heteroskedastic time series model for security prices and rates of return data," The Review of Economics and Statistics, vol. 69, no. 3, pp. 542-547, 1987.

[20] A. K. Nikoloulopoulos, H. Joe, and H. Li, "Extreme value properties of multivariate t copulas," Extremes, vol. 12, no. 2, pp. 129-148, 2009.

[21] R. L. Smith, “Threshold methods for sample extremes," Statistical Extremes and Applications, vol. 131, pp. 621-638, 1985.

[22] A. A. Balkema and L. de Haan, "Residual life time at great age," The Annals of Probability, vol. 2, no. 5, pp. 792-804, 1974.

[23] J. Pickands III, "Statistical inference using extreme order statistics," Annals of Statistics, vol. 3, no. 1, pp. 119-131, 1975.

[24] J. Beirlant, P. Vynckier, and J. L. Teugels, "Excess functions and the estimation of the extreme-value index," Bernoulli, vol. 2, no. 4, pp. 293-318, 1996. 
[25] P. H. Kupiec, "Techniques for verifying the accuracy of risk measurement models," The Journal of Derivatives, vol. 3 , no. 2 , pp. 73-84, 1995.

[26] C. Xie, J. J. Yang, and Y. J. Zhao, "Setting of futures maintenance margin levels based on SV-M-POT-PSRM model: an empirical analysis on high-frequency data of CSI300 stock index futures," Journal of Systems and Management, vol. 22, no. 6, pp. 768-776, 2013 (Chinese). 


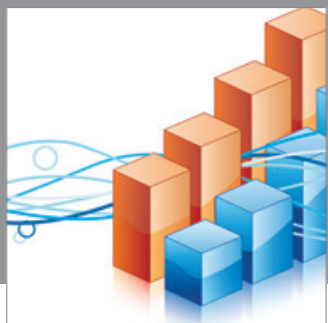

Advances in

Operations Research

mansans

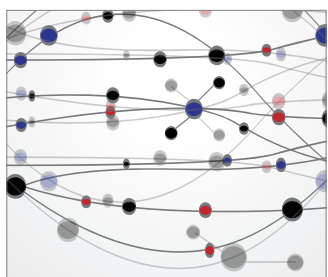

The Scientific World Journal
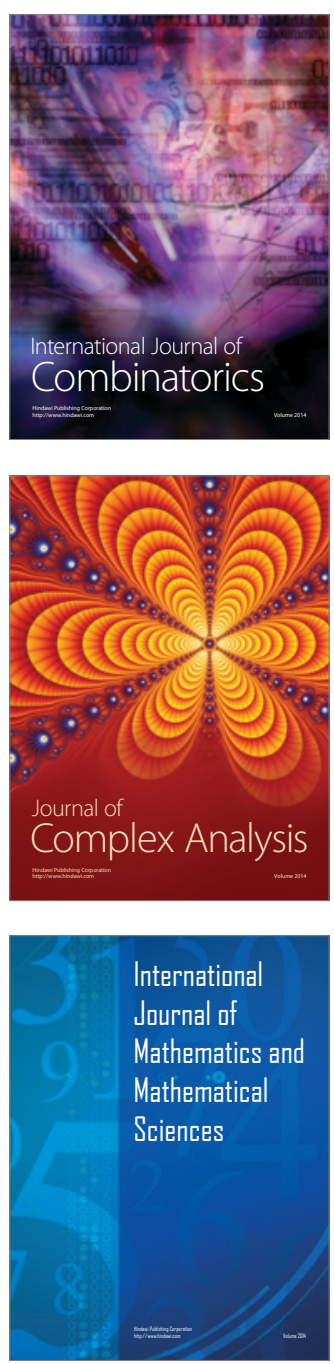
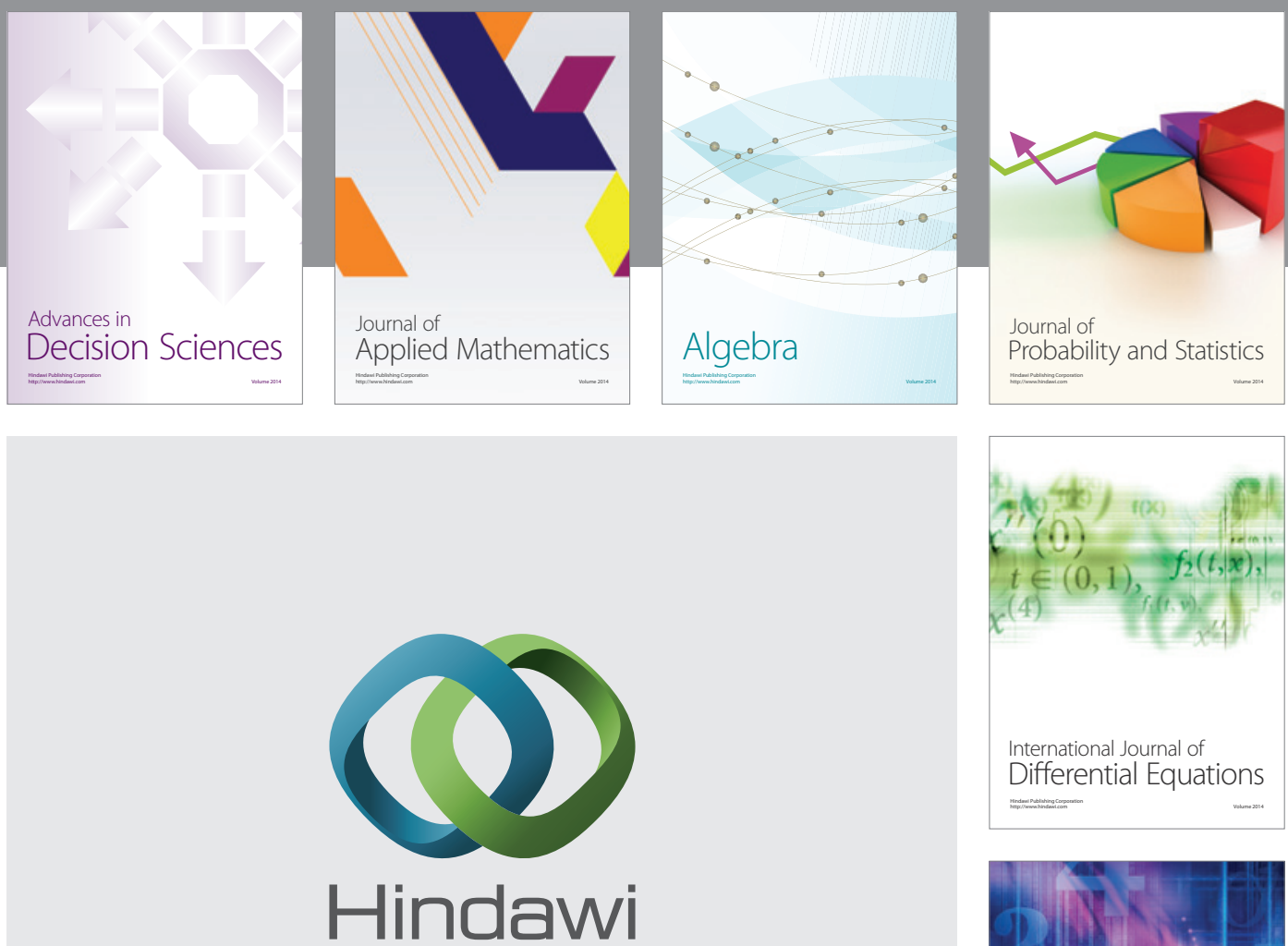

Submit your manuscripts at http://www.hindawi.com
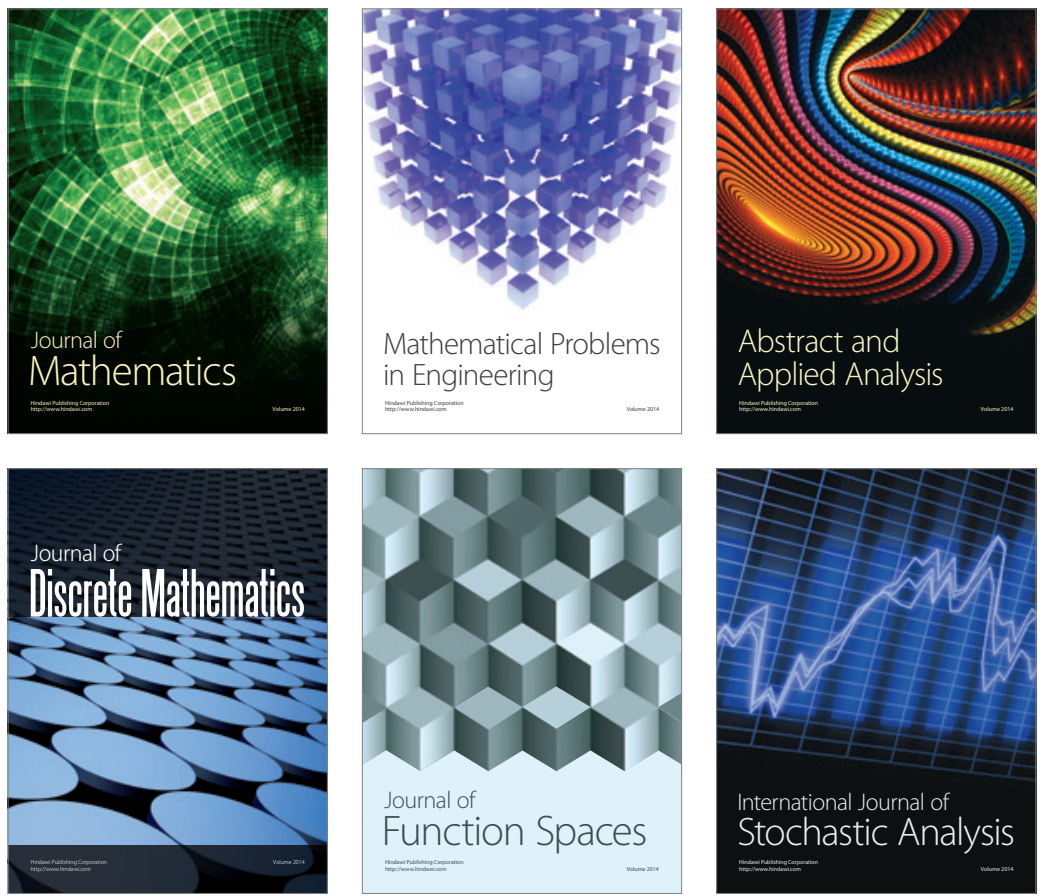

Journal of

Function Spaces

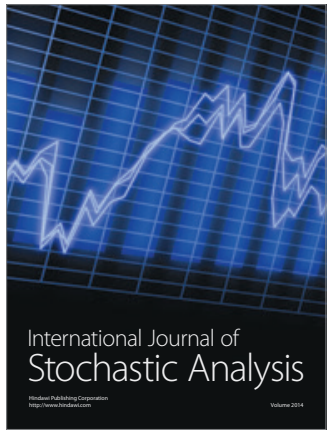

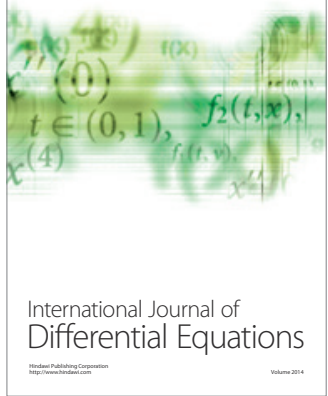
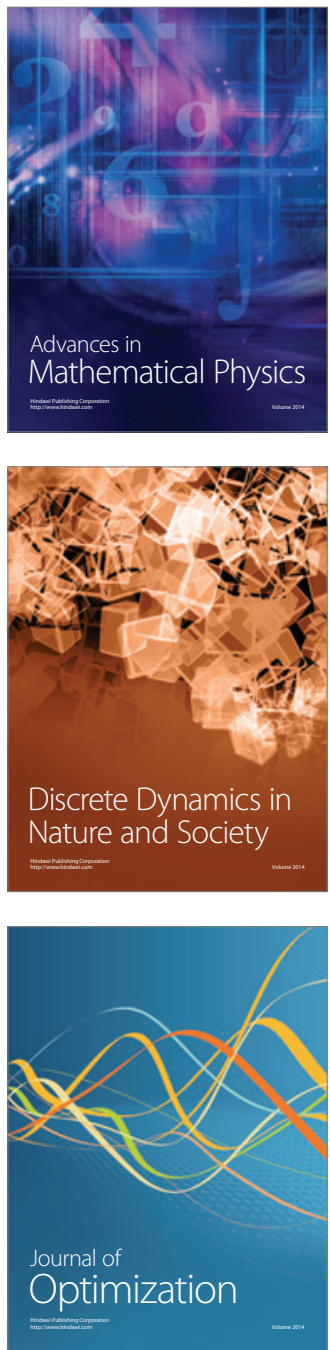